\title{
A potent myeloid response is rapidly activated in the lungs of premature Rhesus
} macaques exposed to intra-uterine inflammation.

Courtney M. Jackson ${ }^{1,2^{*}}$, Martin Demmert ${ }^{1,3^{*}}$, Shibabrata Mukherjee ${ }^{1}$, Travis Isaacs ${ }^{1}$, Jerilyn Gray ${ }^{4}$, Paranthaman Senthamaraikannan ${ }^{4}$, Pietro Presicce ${ }^{5}$, Kashish Chetal $^{6}$, Nathan Salomonis ${ }^{6}$, Lisa A. Miller ${ }^{7}$, Alan H. Jobe ${ }^{4}$, Suhas G. Kallapur ${ }^{5}$, William J. Zacharias $^{4,8}$, Ian P. Lewkowich ${ }^{1}$, Hitesh Deshmukh ${ }^{4}$, Claire A. Chougnet ${ }^{1 \#}$

1. Division of Immunobiology, Cincinnati Children's Hospital Research Foundation, Department of Pediatrics, University of Cincinnati College of Medicine, Cincinnati, Ohio, USA

2. Immunology Graduate Program, University of Cincinnati College of Medicine, Cincinnati, Ohio, USA

3. Department of Pediatrics, Institute for Systemic Inflammation Research, University of Lübeck, Lübeck, Germany

4. Division of Neonatology/Pulmonary Biology, The Perinatal Institute, Cincinnati Children's Hospital Research Foundation, Department of Pediatrics, University of Cincinnati College of Medicine, Cincinnati, Ohio, USA

5. Divisions of Neonatology and Developmental Biology, David Geffen School of Medicine at the University of California Los Angeles, Los Angeles, CA USA

6. Division of Biomedical Informatics, Cincinnati Children's Hospital Research Foundation, Department of Pediatrics, University of Cincinnati College of Medicine, Cincinnati, Ohio, USA

7. California National Primate Research Center, University of California Davis, Davis, CA, Department of Anatomy, Physiology, and Cell Biology, School of Veterinary Medicine, University of California Davis, Davis, CA USA

8. Division of Pulmonary and Critical Care Medicine, Department of Internal Medicine, University of Cincinnati, Ohio, USA

${ }^{*}$ Contributed equally.

\#Corresponding author: Dr. Claire Chougnet, Division of Immunobiology, Cincinnati Children's Hospital Medical Center, MLC \#7038, 3333 Burnet Avenue, Cincinnati, OH 45229-3039. E-mail address: Claire.Chougnet@cchmc.org. phone number: 513-6368847, fax number: 513-636-5355 


\section{Abstract}

Intrauterine inflammation/infection (IUI), which is present in up to $40 \%$ of premature births, leads to elevated levels of pro-inflammatory mediators and microbial products within the amniotic fluid, which come in close contact to fetal mucosae. Yet, knowledge on the fetal mucosal responses to IUI exposure remains limited. To address these questions, we used a non-human primate model of IUI, in which pregnant Rhesus macaques received intraamniotic (IA) LPS, compared with IA saline.

We found that IA LPS exposure induced a robust and rapid inflammation of the fetal lung, but not the intestine. This inflammatory response was characterized by high levels of proinflammatory cytokines in the lung and the alveolar wash, and a potent myeloid cell response, dominated by neutrophils and monocytes/macrophages. scRNAseq analyses of fetal lungs showed that the infiltrating (neutrophils and inflammatory monocytes) and the resident (alveolar and interstitial macrophages) myeloid cells exhibited transcriptional profiles consistent with exposure to TLR ligands, as well as to cytokines, notably IL-1 and TNF $\alpha$. However, blocking IL-1 signaling or TNF $\alpha$, alone or simultaneously by administering inhibitors intra-amniotically and subcutaneously to the dam only partially blunted fetal lung inflammation.

52 Together, our novel data indicate that the fetal innate immune system can mount a rapid multi-factorial mucosal innate response to IUI, responding both to direct signaling by

54 bacterial products and to indirect cytokine-mediated pathways of activation. These data 55 thus provide more mechanistic insights into the association between IUI exposure and the post-natal lung morbidities of the premature infant. 
bioRxiv preprint doi: https://doi.org/10.1101/2021.05.28.444219; this version posted May 29, 2021. The copyright holder for this preprint (which was not certified by peer review) is the author/funder. All rights reserved. No reuse allowed without permission.

57 


\section{Introduction}

Intrauterine inflammation/infection (IUI) is present in $25-40 \%$ of premature births ${ }^{1}$. Inflammation of the placental membranes at the fetal-maternal interface, which is called chorioamnionitis (chorio) $)^{2}$, leads to elevated levels of pro-inflammatory mediators and microbial products within the amniotic fluid ${ }^{2-5}$. Due to in utero aspiration/swallowing of the amniotic fluid ${ }^{6,7}$, the mucosae of the developing fetus come in close contact with these mediators, which triggers an inflammatory response. Although such responses likely contribute to the increased incidence of post-natal pulmonary and intestinal morbidities associated with IUI exposure ${ }^{8-10}$, knowledge about the cells and pathways involved in the fetal mucosal response remains very limited, due to the inability to collect tissue samples in preterm infants.

Fetal lung and intestinal inflammation have been described in IUI animal models (rodent, rabbits, pig, and sheep). Main features include high levels of pro-inflammatory cytokines and infiltration of immune cells, as well as damage to the lung and intestine ${ }^{11-}$ 18. However, the ontogeny of the immune system or the placenta structure are quite different in these species from those in humans. In contrast, non-human primates (NHP) are quite similar to humans in most biological aspects. Furthermore, many reagents developed against human antigens are cross-reactive allowing for in-depth analysis of the NHP fetal immune system ${ }^{19-21}$. Importantly, we have previously shown that intraamniotic (IA) injection of LPS in pregnant Rhesus macaques leads to a type of inflammation that closely phenocopies severe human chorio ${ }^{22,23}$. Previous studies from ours and other groups have reported elevated levels of inflammatory cytokines in the lung of IUI-exposed rhesus macaque fetuses ${ }^{24,23}$, but no in-depth analysis of mucosal immune 
81 responses was performed in these studies. Our goal herein was thus to apply state-of-

82 the art technologies, such as single-cell RNAseq (scRNAseq), to obtain a comprehensive

83 view of the mucosal immune responses occurring in response to IUI.

Furthermore, this model allows us to evaluate the role inflammatory cytokines may play in these responses. Among the cytokines elevated in the amniotic fluid and cord blood in chorio, IL-1 $\beta$ and TNF $\alpha$ appear particularly important ${ }^{4,25}$. Indeed, both cytokines

87 are capable of inducing chorio, and neutrophil infiltration into the fetal lung of Rhesus macaques when administered IA to pregnant dams ${ }^{26,27}$. In addition, IL-1 $\beta$ is involved in many aspects of LPS-induced fetal inflammation $22,23,27-30$. We thus aimed at better delineating their respective role, using the cross-reactive IL-1 receptor antagonist (IL-

91 1RA) and a blocking anti-TNF Ab (Adalimumab) given either individually, or in 92 combination.

Herein, we show that IA LPS leads to rapid and robust inflammation of the fetal

94 lung, but not of the fetal intestine. Notably, our analyses identified two main components,

95 i.e., the recruitment of innate myeloid cells into the fetal lung, and the activation of resident 96 lung myeloid cells. Although signaling by IL-1 and TNF $\alpha$ were identified as major

97 transcriptional signatures in responsive myeloid cells, their individual or combined 98 blockade only partially blunted fetal lung inflammation. Together, our novel data thus 99 indicate that the fetal innate immune system is capable of mounting a rapid multi-factorial 100 mucosal response to IUI, responding both to direct signaling by bacterial products such 101 as LPS, and to indirect cytokine-mediated pathways of activation. 


\section{Results}

IA LPS induces robust inflammation in the fetal lung with a minimal response in the small intestine.

To model IUI, pregnant Rhesus macaques were given intra-amniotic (IA) injection of LPS or saline at approximately $80 \%$ gestation. Sixteen hours post IA injections, the fetuses were surgically removed, and tissues collected for analysis. All the animals included in the study are described in Table S1.

We first examined the histological changes in the fetal lung and small intestine. Compared

111 to controls, lungs from 16hr IA LPS-exposed fetuses displayed signs of inflammation with

112 cell infiltration into the lung interstitium and decreased alveolar space (Fig. 1A).

113 Secondary alveoli septa formation also appeared diminished (Fig. 1A). In contrast, in the 114 jejunum of exposed fetuses, the intestinal villi, lamina propria, or the underlying mucosal 115 area exhibited no abnormalities (Fig. S1A). Because intestinal inflammation did not 116 develop until 3 days after IUI in other models (pig ${ }^{18}$ and sheep ${ }^{12,17,31}$ ), we analyzed

117 jejunum sections taken 48 and 120hrs post IA LPS, and again, found only minimal 118 changes compared to controls (Fig. S1A).

119 Along with histological evaluations, mRNA expression of inflammatory cytokines was 120 measured in lung and jejunum at $16 \mathrm{hrs}$ post IA LPS. TNF $\alpha$, IL-6, and IL-1 $\beta$ mRNA levels 121 were greatly increased in the lung (>100-fold) of IA LPS-exposed fetuses along with 122 increased detection of these cytokines in the alveolar wash (AW) (Fig. 1B, C). Other pro123 inflammatory cytokines such as IL-8 and CCL2 were also increased (see Tables S2 and S3). In contrast, IL-10, a known anti-inflammatory molecule was only minimally increased 
125 in the AW of LPS-exposed infants (Table S2). In agreement with the absence of 126 histological alterations in the jejunum, mRNA expression of inflammatory cytokines was

127 not significantly increased in this compartment (Fig. S1B). Thus, IA LPS induces a rapid 128 inflammatory response within the fetal lung but has minimal impact on the fetal small 129 intestine. Our in-depth analyses of fetal mucosal responses thus focused on the lung.

\section{IA LPS triggers a large infiltration of myeloid cells into the fetal lung.}

We next evaluated by scRNAseq the global transcriptional response of the lung in response to IA LPS (animals and sequencing metrics are described in Table S4). These analyses revealed distinct immune and non-immune cell populations in both control and IA LPS-exposed animals (Fig. 2A, Table S5). As our focus was on immune responses, subsequent analyses were performed on a subset of the whole dataset, containing only the immune cell clusters. (Fig. 2B). Using established lineage marker genes (see Fig. 2C), we identified the robust accrual of neutrophilic and monocyte/macrophage populations in the lung of IA LPS-exposed fetuses, compared to control animals (Fig. 2B). As expected, they highly expressed the myeloid-associated CD88 gene (C5AR1, Fig.

141 2D). These findings were confirmed by flow cytometric analyses of lung cell suspensions

142 (see gating strategy in Fig. S2), which showed a trend toward a higher proportion of $143 \mathrm{CD} 45^{+}$hematopoietic cells, and, within this population, a significant increase in the 144 proportion of $\mathrm{CD}^{+} 8^{+}$cells (Fig. 2E).

145 Due to the rapid response within the lung and the type of challenge used, another myeloid 146 population of interest were dendritic cells (DCs), as DC populations have been described 
147 in the human fetal lung ${ }^{32}$. By flow cytometry, we readily identified myeloid dendritic cells 148 (mDCs) and plasmacytoid DCs (pDCs) in the lung, but their frequencies were unchanged 149 by IA LPS (Fig. S3). However, these DCs appeared activated in IA LPS-exposed fetuses, 150 as they both exhibited significantly increased HLA-DR levels (Fig. S3A-B).

151 In contrast to the brisk myeloid response, the frequency of B cells or different $\mathrm{T}$ cell 152 populations, including regulatory $T$ cells, was not changed by IA LPS as seen by flow 153 cytometry and scRNAseq analyses (Fig. S4A-D). Additionally, analyses of differentially 154 expressed genes (DEGs) also uncovered relatively few changes $(n=342)$ in these 155 populations between IA LPS and control animals (Fig S4B, S4D, Table S6).

156 Taken together, our data show that IA LPS rapidly affected fetal innate immune cell 157 populations in the lung, mainly altering myeloid cells (neutrophils, 158 monocytes/macrophages, DCs), while having little effect on the major lymphoid cell 159 subsets.

IA LPS leads to the recruitment of inflammatory monocytes into the fetal lung and activates the resident macrophages. scRNAseq analyses of the lungs of control fetuses revealed the presence of 2 164 macrophage populations (Fig. 3A), which we identified as alveolar and interstitial 165 macrophages (Fig. 3B and S5). In the lung of IA LPS-exposed fetuses, another population 166 of monocytes/macrophages with characteristics of inflammatory monocytes was also 167 present (Fig. 3A-B and Fig. S5). 
168 Next, we identified DEGs in alveolar and interstitial macrophages of IA LPS exposed

169 lungs compared to controls. The majority of these DEGs were more highly expressed in

170 LPS than in controls $(n=482 / 620$ in alveolar macrophages and $n=302 / 324$ in interstitial

171 macrophages; Table S7, S8). Gene ontology (GO) enrichment analysis of these

172 upregulated genes revealed several biological processes, including activation of the TLR

173 signaling pathway, and response to cytokines, notably to IL-1 and TNF (Fig. 3C, Tables

174 S9 and S10 for full list of genes). Furthermore, genes associated with apoptotic signaling

175 and cell migration were particularly enriched (Fig. 3C). The fewer down-regulated genes

176 in the alveolar and interstitial macrophages of IA LPS lungs mainly included ribosomal

177 protein genes (Tables S6 and S7).

178 There was no inflammatory monocyte cluster in the control lungs, precluding DEG 179 analysis. However, single sample gene set enrichment analysis (ssGSEA) of this 180 population in the IA LPS-exposed lungs revealed a similar profile as that found in the 181 alveolar and interstitial macrophages from the same lungs. Indeed, there was an 182 enrichment of genes related to activation of the TLR, IL-1 and TNF signaling, as well as 183 cell migration and apoptosis (Fig. 3D, Table S11 for full list of genes expressed and 184 enrichment scores). All together, these data thus show that exposure to IUI strongly 185 affects fetal lung resident macrophages as well as rapidly inducing the afflux of activated, 186 inflammatory monocytes into the fetal lung. 
190 Our data also showed a strong neutrophilic response in the fetal lung after IA LPS. 191 scRNAseq analyses identified TNFAIP6 (TNF Alpha Induced Protein 6) expression as 192 one of the most specific markers for neutrophils (Fig. 4A and Fig. S6A). S100A8 was 193 also found to be highly expressed in most neutrophils, although it was also present in the 194 monocyte/macrophage cluster (Fig. 4A). The cluster was further uniquely characterized 195 by the expression of neutrophil specific markers $A Z U 1$, ITGAM, NCF2. By IHC, we 196 confirmed the presence of multiple TNFAIP6 ${ }^{+}$S100A8 ${ }^{+}$cells in the IA LPS fetal lungs, 197 which were not present in control lungs (Fig. 4B). These cells had neutrophilic 198 morphology, and they formed aggregates within alveolar spaces (Fig. 4B-C). We confirmed these data by staining with CD68 and HLA-DR, as we had found that CD68 is highly expressed in both fetal neutrophils and monocytes/macrophages, with only the latter expressing HLA-DR. A significantly increased number of CD68+HLA-DR- cells, with 202 a neutrophilic morphology, were found in the IA LPS-exposed lungs, also forming 203 aggregates (Fig. S6B). Furthermore, we found a significant number of neutrophils in the AW of IA LPS exposed fetuses, whereas they were largely absent in the AW of control 205 fetuses [median 0.0 v. $106.3 \times 10^{6}$ cells $/ \mathrm{kg}$ in control $(n=18)$ and IA-LPS $(n=13)$, 206 respectively, $p<0.0001$, Mann-Whitney test].

We next analyzed the transcriptomic program of the neutrophils recruited in the fetal lungs by ssGSEA. These analyses revealed the enrichment of similar processes as those found in monocytes/macrophages, notably migration, response to TLR, IL-1 and TNF, and 210 apoptosis (Fig.4D and Table S12 for full list of genes expressed and enrichment scores).

211 Interestingly, and in line with other studies ${ }^{33,34}$, substantial transcriptional heterogeneity 212 could be seen within these recruited neutrophils. However, no distinct sub-clusters were 
213 revealed by UMAP projection (Fig. 5A), suggesting different levels of maturation and/or

214 activation. We therefore applied pseudo-time analyses as implemented in Monocle3 to

215 characterize this heterogeneity. Genes that most determined the trajectory were

216 calculated with spatial autocorrelation analysis (Fig. S7). This analysis revealed that

217 cytokine and cytokine-response genes were particularly enriched close to the beginning

218 of the trajectory, whereas expression of other effector genes (e.g., S100A8/9, CAMP,

219 MMP8) was higher towards more advanced pseudo-time (Fig. 5, S7 and S8). Thus, the

220 recruited neutrophils into the fetal lung exhibit transcriptional programs revealing different

221 stages of activation, compatible with both direct activation by LPS and indirect cytokine-

222 mediated pathways.

\section{IL-1 and TNF $\alpha$ signaling are partially driving fetal lung inflammation.}

225 Knowing the role of $\mathrm{IL}-1$ and TNF $\alpha$ in chorio pathogenesis ${ }^{22,23,26-30}$, and because 226 scRNAseq analyses revealed gene signatures consistent with IL-1 and TNF $\alpha$ signaling in 227 both the monocyte/macrophage and neutrophil lung populations of IA LPS animals, we 228 blocked IL-1 signaling with the IL-1RA and TNF $\alpha$ with the blocking anti-TNF Ab 229 Adalimumab, administered both subcutaneous and IA to the pregnant dams. We also 230 tested their combined effect (see Fig. S9 for experimental scheme). Both reagents had 231 previously been shown to be efficacious in macaques $22,23,35$.

232 Cytokine levels in the AW were only partially diminished with any of the treatments (Table 233 S2). This modest effect was confirmed by the fact that cytokine mRNA expression in the 234 lung was also minimally decreased in all the treatment groups (Table S3). LPS-induced 
235 histological changes (cell infiltration and lung interstitium thickening) remained present in 236 animals given IL-1RA or anti-TNF, although lung interstitium thickening was partially 237 decreased in animals given both inhibitors (Fig. 6A).

238 Infiltration of myeloid cells into the lung was not blunted by any of the treatments, as 239 shown by both scRNAseq and flow cytometry analyses (Fig. 6B-C). Neutrophil 240 accumulation in the AW was also not blunted ( $p>0.9$, Kruskal-Wallis tests). Similar 241 monocyte/macrophage populations were present in the lung of treated animals as in IA 242 LPS animals (Fig. 7A). However, the blockades partially suppressed some of the 243 biological processes activated by IA LPS (Fig. 7B and S10A-C). Notably, there was a 244 downward trend of the expression of genes associated with the TLR, IL-1, and TNFa 245 signaling pathways in the alveolar and interstitial macrophages, which appeared most 246 marked in the animals that had received the combined treatment (Fig. 7B). Blockades 247 also decreased the expression of genes associated with cell migration and apoptotic 248 signaling in these cells (Fig. S10A-B). However, the blockades did not blunt these 249 processes in the inflammatory monocytes (Fig. 7B and S10C).

250 Neutrophil migration into the lung was also not diminished by any of the blockades (Fig. 251 8A). As in the inflammatory monocytes, the blocking treatments did not markedly alter the 252 neutrophilic transcriptome (Fig. 8B and S10D).

253 Blockades lead to a decreased frequency of the mDC population, decreasing it below that 254 present in the control animals (Fig. S11). mDC activation, as evidenced by high HLA-DR $255 \mathrm{MFI}$, was diminished by the treatments (Fig. S11). In that case, the strongest effect was 256 seen in animals that received IL-1RA, alone or in combination (Fig. S11). 


\section{Discussion}

258 Premature infants exposed to IUI are at increased risk of developing pulmonary and 259 intestinal morbidities after birth 9,36,37. Herein, we applied several complementary 260 techniques to obtain a comprehensive view of the fetal mucosal immune response to IUI 261 in the highly relevant Rhesus macaque model.

262 In this model, shortly after IA LPS, robust inflammation developed in the fetal lungs, 263 characterized by the accumulation of immune cells into the lung and increased levels of 264 many inflammatory mediators both in the lung tissue and the AW, similar to what had previously been described in other IUI animal models 10,11,28,29. Myeloid cells were 266 particularly involved, as the major cellular change occurring in the lungs of IA LPSexposed fetuses was the massive accrual of neutrophils and inflammatory monocytes, which were absent in the control lungs. In addition, resident myeloid cells, both the alveolar and interstitial macrophages and the resident myeloid DCs, exhibited many signs of activation. Notably, scRNAseq analyses identified that response to pro-inflammatory

271 cytokines, such as IL-1 and TNF, or to TLR, were activated in these subsets. Many of

272 the activated biological processes were shared by the monocyte/macrophage and

273 neutrophil populations, highlighting a general inflammatory transcriptional program.

274 Overall, these data indicate that the fetal innate immune system can rapidly mount a

275 strong response, a concept that remains controversial. Indeed, there have been many 276 papers describing functional impairment in fetal/neonatal neutrophils and 277 monocytes/macrophages as compared to their juvenile and adult counterparts (rev. in ${ }^{38-}$ $27{ }^{40}$ ). Described defects notably include the decreased recruitment of neutrophils and 279 inflammatory monocytes into the lung in response to $E$. coli instillation during the same 
280 time period, compared to juvenile mice ${ }^{41}$. In contrast, our data in a NHP model argue that 281 fetal neutrophils and inflammatory monocytes can quickly migrate into the pulmonary

282 environment, as these two populations became very abundant in the lung 16hrs after IA

283

284

285

286

287

288

289

290

291

292

293

294

295

296

297

298

299

300

301

302

LPS. Neutrophils appeared to localize in the lung in the form of aggregates, a pattern reminiscent of that described in murine models of acute lung injury ${ }^{42,43}$. Similarly, other groups had described rapid recruitment of neutrophils in fetal sheep or rhesus macaque lungs in response to IA LPS or IL-1 ${ }^{27,28}$. It is also to be noted that the concept of defective functionality of innate immune cells in human neonates mainly arises from studying in vitro stimulated cord blood cells. It is therefore possible that these assays do not fully reveal their potential. Another caveat to remember when interpreting data about preterm babies' immune cell functions, is that many of these infants had just been given steroids, which affect various cell type functionality. We did not administer steroids in our studies, which may have allowed us to observe such robust immune responses.

Until recently, circulating and tissue neutrophils were considered as a homogeneous population with a defined array of functions. This concept has been challenged by many studies that revealed a high degree of variety in this population (rev. in ${ }^{44-49}$ ). Two main mechanisms appear to drive this diversity, an intrinsic heterogeneity already initiated in bone marrow precursors on one hand, and on the other hand a highly dynamic transcriptional and epigenetic program triggered by exposure to the environment, notably in inflammatory conditions. Our pseudo-time analyses concur with the concept of functional heterogeneity, as they show several gene expression profiles within the neutrophils recruited to the lungs of IA LPS exposed animals. For example, genes related to granule content (CAMP, LCN2) or antimicrobial defense (S100A8, S100A9) were in 
general not simultaneously expressed along inflammatory mediators (IL1A, IL1B, CCL2, CCL20). Interestingly, Bc/2A1, a major pro-survival factor for neutrophils ${ }^{50}$, including for LPS-activated neutrophils in the Rhesus chorio-decidua ${ }^{22}$, was also heterogeneously expressed among the lung neutrophils in LPS-exposed animals, suggesting that differential survival may affect neutrophil heterogeneity. Future studies will be needed to further characterize at the protein level neutrophil heterogeneity and its impact on fetal responses to IUI.

We were also able to evaluate the role played by IL-1 or TNF $\alpha$ in fetal mucosal responses to IA LPS. Our data show that blocking either or both of these pathways had only a partial

312 effect on lung inflammation, a finding consistent with the fact that fetal responses were 313 likely driven by both direct activation by LPS and indirect cytokine-mediated pathways.

314 The partial amelioration of disease by IL-1RA is similar to what has been previously 315 described in other IUI models $22,23,28,30$, while this was, to our knowledge, the first attempt at evaluating the effect of anti-TNF alone or in combination with IL-1RA. In general, the anti-TNF Ab appeared to have broader effectiveness than IL-1RA, which may be due to

318 the fact that this treatment decreased levels of IL-1 expression in the lung tissue and AW, whereas IL-1RA did not affect TNFa levels (Tables S2-S3). Interestingly, the combination of both blockades did not radically ameliorate the effectiveness, which suggests that the two pathways are inter-connected, and that other mediators control the

322 recruitment of myeloid cells into the lung. Notably, levels of CCL2, which regulates many 323 aspects of monocyte biology, including recruitment, activation, and survival (rev. in ${ }^{51}$ ), 324 remained elevated in the lung tissue and AW of treated animals. Lung expression of 
326 be involved. Our data also show that high levels of IL-8 persisted in the lung and AW

327 despite the blockades (Tables S2 and S3). Furthermore, many neutrophilic

328 chemoattractant molecules, including chemotactic lipids, formyl peptides, and

329 complement anaphylatoxins are also released during inflammation (rev. in ${ }^{54}$ ). We did not

330 quantify these mediators in fetal lungs or AW, but because they are released through both

331 TLR and cytokine-mediated mechanisms, it is likely that they were still released despite

332 the blockades. Interestingly, compared to the fetal lung, myeloid cell recruitment in the

333 placenta was significantly reduced in the presence of either IL-1RA or anti-TNF 22,35,

334 suggesting that the mechanisms controlling cellular migration might be tissue specific.

335 Alternatively, and not exclusively, the fact that cells recruited in the placenta have a

336 maternal origin, in contrast to fetal cells migrating to the lungs, may also have driven some

337 of these differences.

Overall, our work in a model that phenocopies the main features of human IUI gives novel insights into the inflammation rapidly developing in the fetal lung following such exposure. responsive to its environment. In this model of acute sterile inflammation, we saw that fetal myeloid cells could be rapidly mobilized, migrating to the inflamed environment and displaying profiles of activation very similar to those described in adult cells. This confirms

344 the new paradigm in the field of neonatal immunology, e.g., that fetuses have the capacity to mount a very robust innate immune response. Our data also emphasize the fact that

346 the fetal lung is particularly responsive, as it is in close contact with the amniotic fluid, 347 whereas the fetal gut appears less affected. This study thus provides more insights into why the most solid associations found between IUI exposure and post-natal morbidities 
349 in humans involved mainly the lung (bronchopulmonary dysplasia, wheeziness, and 350 asthma). Our data also show that the multi-parametric mucosal inflammation that 351 develops in IUI-exposed fetuses is not easily controlled by blocking only one, or two 352 inflammatory mediators, emphasizing the need for additional mechanistic studies to better 353 understand the dynamic and complex responses that IUI triggers in exposed fetuses. 
Materials and Methods.

368

369

370

371

372

373

374

375

376

377

378

379

380

381

382

383

\section{Animals.}

The Institutional Animal Care and Use Committee at the University of California, Davis approved all animal procedures. Normally cycling, adult female Rhesus macaques (Macaca mulatta) were time mated. At approximately 130 days of gestation (about $80 \%$ of term gestation), the pregnant dams received either $1 \mathrm{ml}$ saline solution or $1 \mathrm{mg}$ of LPS (Sigma-Aldrich, St. Louis MO) in $1 \mathrm{ml}$ saline solution by ultrasound guided IA injection. IA administration of LPS or saline was performed in mothers of similar weights and ages with fetus with similar fetal genders and gestational ages (Table S1). Fetuses were surgically delivered 16,48 or 120 hrs later by cesarean section. Delivered fetuses were euthanized with pentobarbital and tissues collected. There were no spontaneous deaths or preterm labor in the animals. Some pregnant dams were given, in addition to IA LPS, either the IL-1RA (Kineret $®$ Sobi, Stockholm, Sweden), or the anti-TNF Ab Adalimubab (HUMIRA® AbbVie Chicago, IL), or both. These inhibitors were administered subcutaneously (100mg or 40mg for IL-1RA and anti-TNF respectively) 3hrs before IA LPS as well as IA (50mg or $40 \mathrm{mg}$ for IL-1RA and anti-TNF respectively) $1 \mathrm{hr}$ before IA LPS (see scheme on Fig. S9).

\section{Histological evaluation of fetal lung and jejunum.}

Inflated lung $(30 \mathrm{~cm}$ water pressure) and jejunum tissues were fixed in $10 \%$ formalin immediately following removal. After a series of alcohol and xylene washes, tissues were blocked in paraffin. Paraffin sections were stained with hematoxylin \& eosin (H\&E). 20x images were taken with a Nikon 90i Upright Widefield Microscope (Nikon Instruments 


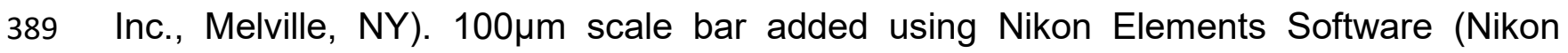

390 Instruments Inc., Melville, NY).

391 Immunofluorescence staining of neutrophils and confocal analysis.

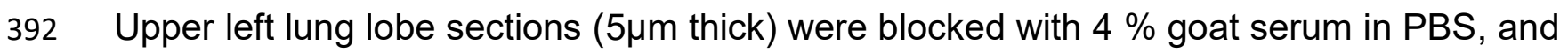
393 then incubated $1 \mathrm{hr}$ at room temperature with the primary mouse-anti-human Ab diluted in $3944 \%$ goat serum in PBS. Cross-reactivity of all Ab with Rhesus macaque cells was verified 395 on adult tissues prior to their utilization to stain fetal cells. Ab were titrated for optimal 396 detection of positive populations and MFI. Sections stained with unlabeled Ab were 397 stained with a corresponding secondary goat-anti-mouse Ab conjugated with either Alexa 398 Fluor 594, Alexa Fluor 488 or FITC (Life Technologies, dilution 1:400) for $1 \mathrm{hr}$. As negative 399 control, tissues were also stained with the secondary Ab only. Sections were washed and 400 incubated with Vecta shield autofluorescence reduction kit and mounted with mounting 401 media containing DAPI (Vector Laboratories). Images were acquired using an inverted 402 microscope (Ti-E; Nikon, Japan) outfitted with a confocal scan-head (A1R; Nikon; Japan) 403 at the CCHMC Confocal Imaging Core. Analyses involving deconvolution and background 404 reduction were done with Nikon Elements Software.

405 Since neutrophils in the sections were mostly in clumps/aggregates, we evaluated the 406 number of neutrophil aggregates in 10 fields per animal. The neutrophils were identified 407 as S100A8-FITC (CF-145) and TNFAIP6-AF594 (polyclonal) (both ThermoFisher 408 Waltham, MA) double positive cells. The average area covered by neutrophils clumps 409 were also determined and normalized to the total DAPI area of the image per field. CD68410 FITC (KP1) (Alligent Dako, Santa Clara, CA) and HLADR-AF594 (L243) (BioLegend, San 411 Diego, CA) were also used to quantify neutrophils. 


\section{Alveolar wash analyses.}

413 Alveolar wash was collected from the left lung. Supernatant was collected and frozen 414 until cytokine analyses, using the NHP multiplex kit according to the manufacturer's

415 protocol (Millipore Austin, TX). Cell pellets was cytospun and slides were stained with

416 Diff quick. 5 fields/slide (100 cells/field) were counted twice.

\section{Quantitative RT-PCR analyses of mucosal tissues.}

418 From frozen lung and jejunum tissue, total RNA was extracted after homogenizing in

419 TRIzol (Invitrogen, Carlsbad, CA). A Nanodrop spectrophotometer (Thermo Fisher 420 Scientific) was used to measure RNA concentration and quality. $1 \mu \mathrm{g}$ of RNA was used to 421 generate cDNA using the Verso cDNA synthesis kit (Thermo Fisher Scientific), following 422 the manufacturer's protocol. Quantitative RT-PCR was done with a StepOnePlus real423 time PCR system and rhesus specific TaqMan gene expression primers (Life 424 Technologies, Carlsbad, CA). Eukaryotic 18S rRNA was the endogenous control used for normalization of the target RNAs. The mRNA fold change was calculated relative to the 426 average value of the control group.

\section{Fetal lung cell isolation.}

428 The fetal lung was removed, and left lobe was processed into single cell suspensions as 429 described previously ${ }^{55}$. The large airways were removed and approximately $\sim 500 \mathrm{mg}$ of 430 airway free tissue was collected in a gentleMACS C-tube (Miltenyi Biotec, Auburn, CA) 431 and $5 \mathrm{~mL}$ digest buffer was added and placed on a gentleMACS octo dissociator for 432 programmed runs. Following dissociation, the cell suspension was passed through a $433100 \mu \mathrm{m}$ filter and washed with PBS. After washing, cells underwent red cell lysis 
434 (eBioscience, San Diego, CA) along with another pass through a 40um filter after 435 neutralization.

436 Single cell sequencing of fetal lung cells.

437 scRNA-seq analyses were done in a subset of animals (Table S4). $\sim 50,000$ cells per lung 438 were submitted for single cell sequencing at the Cincinnati Children's Hospital Medical 439 Center DNA Sequencing and Genotyping Core. Approximately 16,000 cells were loaded 440 into one channel of the Chromium system using the 3 prime v3 single cell reagent kit (10X 441 Genomics, Pleasanton, CA). Following capture and lysis, cDNA was synthesized and 442 amplified as per the manufacturer's protocol (10X Genomics). The amplified cDNA was 443 used to construct Illumina sequencing libraries that were each sequenced using an 444 Illumina HiSeq 4000 machine. Raw sequencing data was processed aligned to the 445 Rhesus macaque reference Mmul_8.0.1 (Ensembl version 91) with Cell Ranger 3.0.2 446 (10X Genomics) generating expression count matrix files.

447 After doublet removal with the software DoubletFinder (version 2.0), an integrated 448 analysis was performed using $\mathrm{R}$ (version 3.6.3) and the Seurat package (version 3.1.0, 449 https://satijalab.org/seurat/), to identify common cell types across different experimental 450 conditions. Specifically, cells with $25 \%$ or more mitochondrial transcripts were removed, 451 as well as cells expressing fewer than 150 or more than 5000 features. After log452 normalization, 5000 highly variable features were identified per sample using the vst 453 method. Integration was performed using the Seurat FindlntegrationAnchors and 454 IntegrateData functions with default parameter values. The integrated data was scaled, 455 including regressing out mitochondrial percentage and cell cycle variables, followed by 
456 application of PCA reduction. A UMAP projection was estimated based on the PCA 457 reduction and the correlation metric. Clustering of cells was done using the Seurat 458 functions FindNeighbors (based on first 30 PCs) and FindClusters (resolution=4). This 459 high-resolution was initially used to define highly discrete immune populations, with the final populations subsequently aggregated into broader, higher confidence cell-types based common marker genes expressed and corresponding UMAP embeddings. To 462 annotate these cell-populations, were performed a gene-set enrichment analysis on the 463 aggregated cell populations used established human and mouse cell-population marker 464 genes annotated by LungMAP, from the AltAnalyze and ToppCell databases. Immune cell population labels were further evidenced using label transfer with the software 466 cellHarmony against a recently described reference human lung datasets ${ }^{56,57}$. Clusters 467 were orthogonally validated using the software ICGS2 in AltAnalyze, which does not 468 require a resolution setting, to confirm their presence. Identified immune cell clusters were 469 subset into a new Seurat object and re-clustered. For further analyses, Y-chromosomal 470 genes were removed from the dataset because both IA LPS-exposed fetuses were male 471 whereas animals of both sexes were present in the other experimental groups.

472 Additionally, we checked for the expression of genes XIST and TSIX, which did not 473 appear in our dataset and seem to not be drivers of differences observed. All identified 474 cell populations were required to have unique reported marker genes to ensure they were 475 not artifacts of "over-clustering". Differential gene expression analysis was performed as 476 implemented in Seurat. Plots were generated using the ggplot2 package (version 3.3.2). 477 Differentially expressed genes (DEGs) between control and LPS in the 478 monocyte/macrophage cluster with a fold change (FC) of $\geq 1.2$ and adjusted $p$-value of $\leq$ 
4790.05 were used for functional enrichment analysis of biological processes and pathways 480 using the ToppFun web portal ${ }^{58}$. Terms with higher expression in LPS are represented 481 as positive log p-values. Scaled expression of genes for upregulated biological processes 482 terms in the monocyte/macrophage cluster were used to plot parallel coordinate plots 483 using the ggplot2 package.

484 Differential gene expression analysis was not feasible for the neutrophil or inflammatory 485 monocyte clusters since those cells were not present in control samples (<20 cells). We 486 thus performed single sample gene set enrichment analysis (SSGSEA) in R using the 487 ssgsea2.0 package (github.com/broadinstitute/ssGSEA2.0) in order to identify enriched 488 gene ontology (GO) gene sets (MSigDB v7.0). Expression values of overlapping genes 489 of the most significant and relevant gene sets were plotted as parallel coordinate plots $490 \quad$ using ggplot2.

491 In order to assess possibly different states of gene expression within neutrophils after 492 LPS exposure, single-cell trajectory analysis was performed with Monocle3 (version 493 0.2.3.0). Spatial autocorrelation analysis as implemented in Monocle3 was used to 494 determine genes that most strongly vary along the pseudo-time trajectory. The Monocle 495 algorithm is capable of ordering cells based on their transcriptomic profile in an 496 unsupervised manner and thus arrange cells along a directional path. The order of the 497 cells along this path represents different transcriptomic states within a biological process 498 even if cells are obtained at only one time point during an experiment. The scRNA-seq 499 data have been deposited in the Gene Expression Omnibus (GSE169390, reviewer 500 token:qxatqgkyhxyrhkl). 
501 Flow Cytometry.

502 A cocktail of conjugated Ab was used to phenotype lung cell suspensions for multi-

503 parameter flow-cytometry. The following Ab were used: HLA-DR (L243), CD11c (3.9)

504 (BioLegend, San Diego, CA); CD3 (GHI/61), CD3 (SP34-2), CD19 (SJ25C1), CD20

505 (2H7), CD123 (7G3), CD45 (DO58-1283) (BD Biosciences, San Jose, CA); CD8a (RPA-

506 T8), FoxP3 (PCH101), LIVE/DEAD Fixable Aqua dead cell stain, (eBioscience, San

507 Diego, CA), CD88 (P12/1) (Bio-rad, Hercules, CA). Lung cell suspensions were treated

508 with human IgG to block Fc-receptors and stained for surface markers. Cells were then

509 washed and treated with fixation/permeabilization buffer (ThermoFisher). Following

510 permeabilization, cells were stained for intracellular markers, washed, and resuspended

511 in PBS. Cells were collected on a FACS Fortessa (BD Bioscience San Jose, CA). BD

512 FACSDiva Software v8.0.1 (BD Bioscience San Jose, CA) was used for analysis.

513 Statistical analyses.

514 GraphPad Prism 8 (GraphPad Software, La Jolla, California, USA) was used to graph

515 and analyze data for statistical significance. Data were first checked for normality and

516 values expressed as either mean \pm SEM or median and interquartile range. Statistical

517 differences between 2 groups were analyzed using Mann-Whitney U-tests or Student's t-

518 test. For comparison of more than two groups, Kruskal-Wallis or One-way ANOVA were

519 used. Results were considered significantly different for $p$ values $\leq 0.05$. However, due to

520 the limited number of samples in some groups, we also report trends ( $p$ values between

5210.05 and 0.1$)$.

522 Author contributions. 
523 C.A.C, A.H.J., H.D., S.G.K., L.A.M., I.P.J. and W.J.Z. conceived the study. C.M.J., S.M.,

524 P.P., T.I., P.S., M.C., M.D. and J.G. all performed experiments and data analysis. N.S.

525 and K.C. did the initial pre-processing of scRNAseq 10X files. All authors critically

526 reviewed the manuscript and approved the final version.

527 Acknowledgments.

528 The authors would like to thank the staff at the California National Primate Research

529 Center for their outstanding technical support, and especially Paul-Michael Sosa, Jennifer

530 Kendrick, and Sarah Davis and for invaluable help in animal management and care. We

531 also thank Cincinnati Children's Hospital Medical Center's Research Flow Cytometry,

532 Confocal Imaging, and DNA Sequencing and Genotyping Cores.

534 Grant funding: This work was funded by NIH (U01ES029234, K08HD084686,

535 R01HL142708, K08HL140178-01A1) and CCHMC (Academic and Research Committee

536 Grant).

537

538

539

540

541 


\section{References}

5441 Goldenberg, R. L., Culhane, J. F., lams, J. D. \& Romero, R. Epidemiology and causes of preterm birth. The Lancet 371, 75-84, doi:10.1016/S0140-6736(08)60074-4 (2008).

$5462 \mathrm{Kim}, \mathrm{C}$. J. et al. Acute chorioamnionitis and funisitis: definition, pathologic features, and clinical 547 significance. Am J Obstet Gynecol 213, S29-S52, doi:10.1016/j.ajog.2015.08.040 (2015).

5483 Saji, F. et al. Cytokine production in chorioamnionitis. Journal of Reproductive Immunology 47, 185-196, doi:https://doi.org/10.1016/S0165-0378(00)00064-4 (2000). Revello, R., Alcaide, M. J., Dudzik, D., Abehsera, D. \& Bartha, J. L. Differential amniotic fluid cytokine profile in women with chorioamnionitis with and without funisitis. The Journal of Maternal-Fetal \& Neonatal Medicine 29, 2161-2165, doi:10.3109/14767058.2015.1077512 (2016). Gomez, R. et al. The fetal inflammatory response syndrome. American Journal of Obstetrics \& Gynecology 179, 194-202, doi:10.1016/S0002-9378(98)70272-8 (1998). protein in the human conceptus. American Journal of Obstetrics \& Gynecology 113, 632-645, doi:10.1016/0002-9378(72)90632-1 (1972).

559 ROSS, M. G. \& NIJLAND, M. J. M. Fetal Swallowing: Relation to Amniotic Fluid Regulation. Clinical Obstetrics and Gynecology 40, 352-365 (1997).

5618 Jobe, A. H. Effects of chorioamnionitis on the fetal lung. Clin Perinatol 39, 441-457,

562

563 doi:10.1016/j.clp.2012.06.010 (2012). Meta-Analysis. The Journal of Pediatrics 162, 236-242.e232, doi:10.1016/j.jpeds.2012.07.012 (2013).

10 Kallapur, S. G., Presicce, P., Rueda, C. M., Jobe, A. H. \& Chougnet, C. A. Fetal immune response to chorioamnionitis. Semin Reprod Med 32, 56-67, doi:10.1055/s-0033-1361823 (2014).

11 Kallapur, S. G., Willet, K. E., Jobe, A. H., Ikegami, M. \& Bachurski, C. J. Intra-amniotic endotoxin: chorioamnionitis precedes lung maturation in preterm lambs. American Journal of PhysiologyLung Cellular and Molecular Physiology 280, L527-L536, doi:10.1152/ajplung.2001.280.3.L527 (2001). exposure of inflammatory mediators or by lung inflammation. Am J Physiol Gastrointest Liver Physio/ 306, G382-G393, doi:10.1152/ajpgi.00260.2013 (2014). alveolarisation. European Respiratory Journal 32, 1520-1528, doi:10.1183/09031936.00023708 (2008).

Pan, J. et al. Effects and molecular mechanisms of intrauterine infection/inflammation on lung development. Respir Res 19, 93-93, doi:10.1186/s12931-018-0787-y (2018). Wolfs, T. G. A. M. et al. Endotoxin induced chorioamnionitis prevents intestinal development during gestation in fetal sheep. PLoS One 4, e5837-e5837, doi:10.1371/journal.pone.0005837 (2009).

58416 Wolfs, T. G. A. M. et al. Antenatal ureaplasma infection impairs development of the fetal ovine gut in an IL-1-dependent manner. Mucosal Immunology 6, 547-556, doi:10.1038/mi.2012.97 (2013). 

Xie, X. et al. Single-cell transcriptome profiling reveals neutrophil heterogeneity in homeostasis and infection. Nature Immunology 21, 1119-1133, doi:10.1038/s41590-020-0736-z (2020). Evrard, M. et al. Developmental Analysis of Bone Marrow Neutrophils Reveals Populations Specialized in Expansion, Trafficking, and Effector Functions. Immunity 48, 364-379.e368, doi:10.1016/j.immuni.2018.02.002 (2018). Presicce, P. et al. TNF-Signaling Modulates Neutrophil-Mediated Immunity at the Feto-Maternal Interface During LPS-Induced Intrauterine Inflammation. Frontiers in Immunology 11, doi:10.3389/fimmu.2020.00558 (2020). Metcalfe, A., Lisonkova, S., Sabr, Y., Stritzke, A. \& Joseph, K. S. Neonatal respiratory morbidity following exposure to chorioamnionitis. BMC Pediatr 17, 128-128, doi:10.1186/s12887-0170878-9 (2017). Lau, J. et al. Chorioamnionitis with a fetal inflammatory response is associated with higher neonatal mortality, morbidity, and resource use than chorioamnionitis displaying a maternal inflammatory response only. American Journal of Obstetrics \& Gynecology 193, 708-713, doi:10.1016/j.ajog.2005.01.017 (2005).

Kumar, S. K. M. \& Bhat, B. V. Distinct mechanisms of the newborn innate immunity. Immunology Letters 173, 42-54, doi:https://doi.org/10.1016/i.imlet.2016.03.009 (2016).

Lawrence, S. M., Corriden, R. \& Nizet, V. Age-Appropriate Functions and Dysfunctions of the Neonatal Neutrophil. Frontiers in Pediatrics 5, doi:10.3389/fped.2017.00023 (2017).

Yu, J. C. et al. Innate Immunity of Neonates and Infants. Frontiers in immunology 9, 1759-1759, doi:10.3389/fimmu.2018.01759 (2018).

McGrath-Morrow, S. A. et al. The innate immune response to lower respiratory tract E. Coli infection and the role of the CCL2-CCR2 axis in neonatal mice. Cytokine 97, 108-116, doi:10.1016/j.cyto.2017.06.002 (2017).

Park, l. et al. Neutrophils disturb pulmonary microcirculation in sepsis-induced acute lung injury. Eur Respir J 53, 1800786, doi:10.1183/13993003.00786-2018 (2019).

Zarbock, A., Singbartl, K. \& Ley, K. Complete reversal of acid-induced acute lung injury by blocking of platelet-neutrophil aggregation. J Clin Invest 116, 3211-3219, doi:10.1172/JCI29499 (2006).

Filep, J. G. \& Ariel, A. Neutrophil heterogeneity and fate in inflamed tissues: implications for the resolution of inflammation. American Journal of Physiology-Cell Physiology 319, C510-C532, doi:10.1152/ajpcell.00181.2020 (2020).

Silvestre-Roig, C., Hidalgo, A. \& Soehnlein, O. Neutrophil heterogeneity: implications for homeostasis and pathogenesis. Blood 127, 2173-2181, doi:10.1182/blood-2016-01-688887 (2016).

$46 \mathrm{Ng}$, L. G., Ostuni, R. \& Hidalgo, A. Heterogeneity of neutrophils. Nature Reviews Immunology 19, 255-265, doi:10.1038/s41577-019-0141-8 (2019).

7 Alder, M. N. et al. Olfactomedin 4 marks a subset of neutrophils in mice. Innate Immun 25, 2233, doi:10.1177/1753425918817611 (2019).

$\mathrm{Hu}, \mathrm{N}$. et al. Coexpression of CD177 and membrane proteinase 3 on neutrophils in antineutrophil cytoplasmic autoantibody-associated systemic vasculitis: Anti-proteinase 3mediated neutrophil activation is independent of the role of CD177-expressing neutrophils. Arthritis \& Rheumatism 60, 1548-1557, doi:10.1002/art.24442 (2009). Pillay, J. et al. A subset of neutrophils in human systemic inflammation inhibits T cell responses through Mac-1. J Clin Invest 122, 327-336, doi:10.1172/JCI57990 (2012).

Vier, J., Groth, M., Sochalska, M. \& Kirschnek, S. The anti-apoptotic Bcl-2 family protein A1/Bfl-1 regulates neutrophil survival and homeostasis and is controlled via PI3K and JAK/STAT signaling. Cell Death Dis 7, e2103-e2103, doi:10.1038/cddis.2016.23 (2016). 
68351 Gschwandtner, M., Derler, R. \& Midwood, K. S. More Than Just Attractive: How CCL2 Influences Myeloid Cell Behavior Beyond Chemotaxis. Frontiers in Immunology 10, doi:10.3389/fimmu.2019.02759 (2019). Petty, J. M. et al. Pulmonary Stromal-Derived Factor-1 Expression and Effect on Neutrophil Recruitment during Acute Lung Injury. The Journal of Immunology 178, 8148-8157, doi:10.4049/jimmunol.178.12.8148 (2007). receptors CXCR4 and CXCR7 attenuates acute pulmonary inflammation via the adenosine $A(2 B)$ receptor on blood cells. Cell Death Dis 8, e2832-e2832, doi:10.1038/cddis.2016.482 (2017).

692 Metzemaekers, M., Gouwy, M. \& Proost, P. Neutrophil chemoattractant receptors in health and disease: double-edged swords. Cell Mol Immunol 17, 433-450, doi:10.1038/s41423-020-0412-0

695 (2020).

696

697 Zacharias, W. \& Morrisey, E. Isolation and culture of human alveolar epithelial progenitor cells.

55 Zacharias, W. \& Mor

698

56 Travaglini, K. J. et al. A molecular cell atlas of the human lung from single-cell RNA sequencing. Nature 587, 619-625, doi:10.1038/s41586-020-2922-4 (2020).

57 DePasquale, E. A. K. et al. cellHarmony: cell-level matching and holistic comparison of single-cell transcriptomes. Nucleic Acids Res 47, e138, doi:10.1093/nar/gkz789 (2019). doi:10.1093/nar/gkp427 (2009). 
Fig. 2

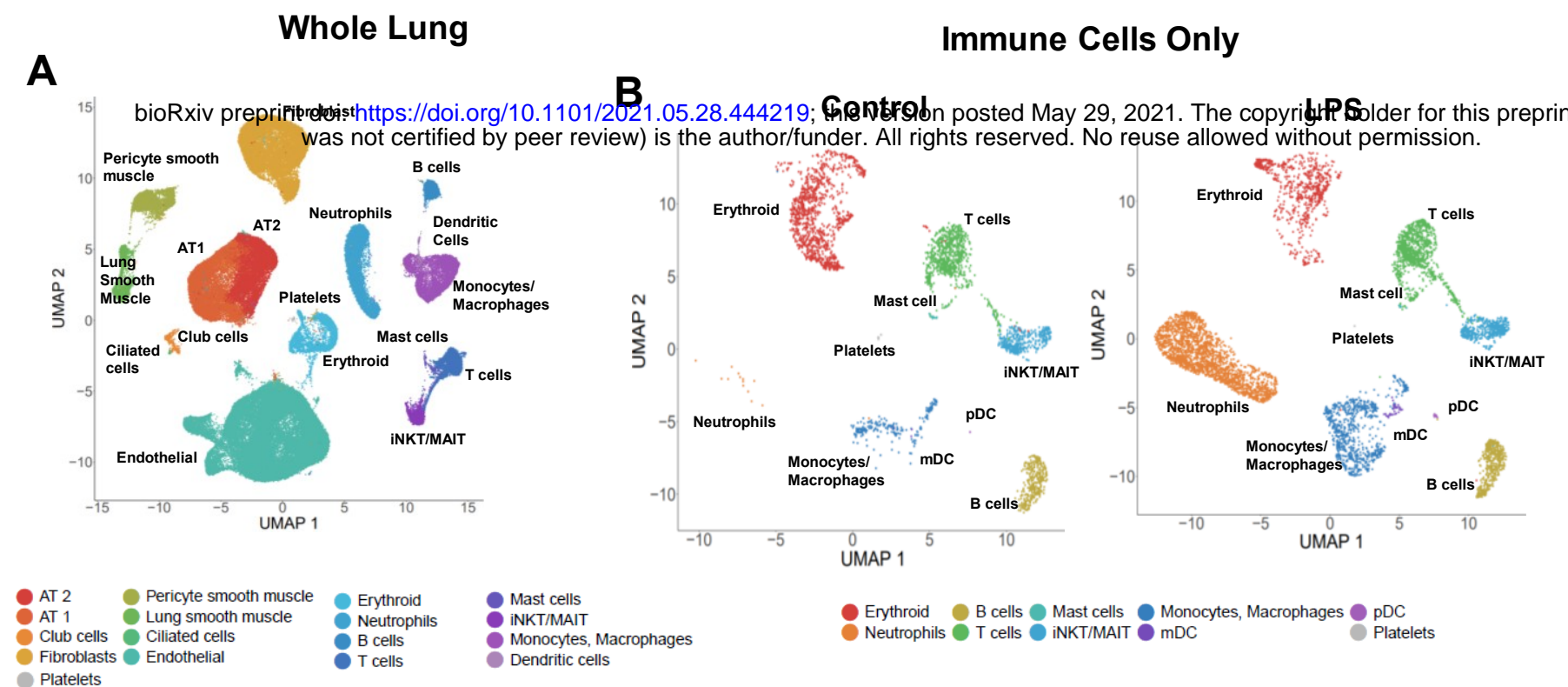

Erythroid B cells Mast cells Monocytes, Macrophages pDC Platelets

C

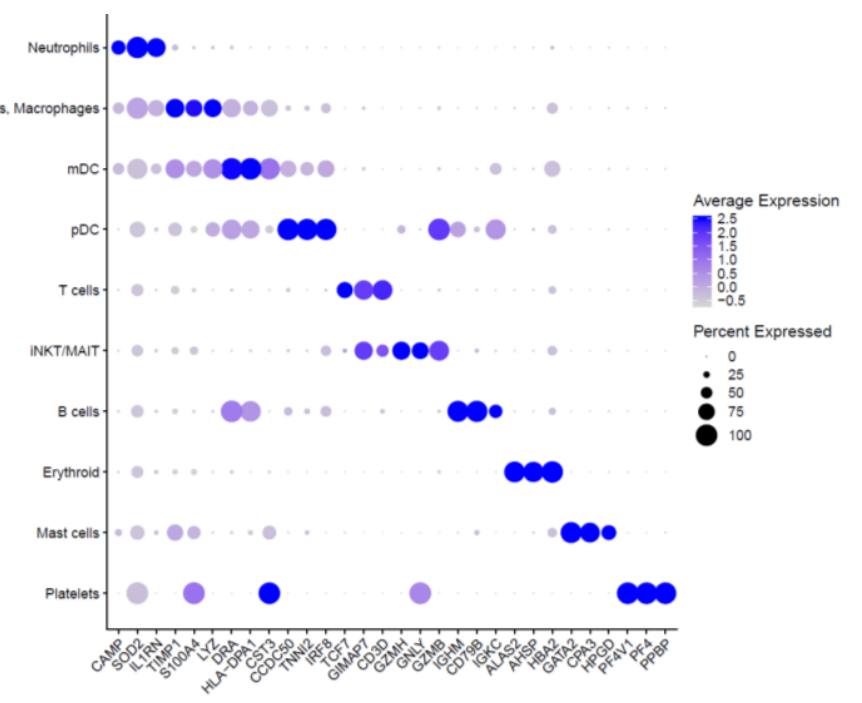

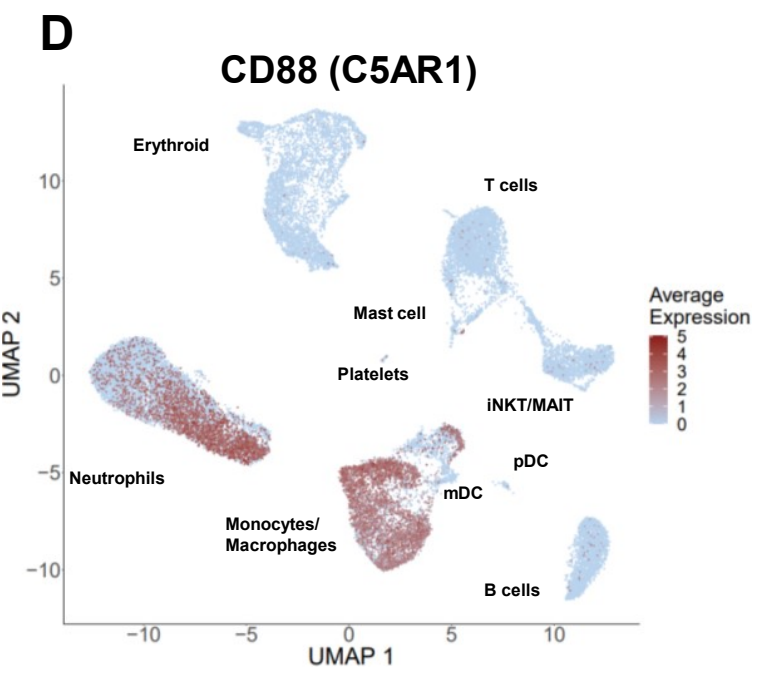

$\mathbf{E}$

CD45 $\%$

$\mathrm{CD}^{2} 8^{+}\left(\mathrm{CD} 45^{+}\right) \%$
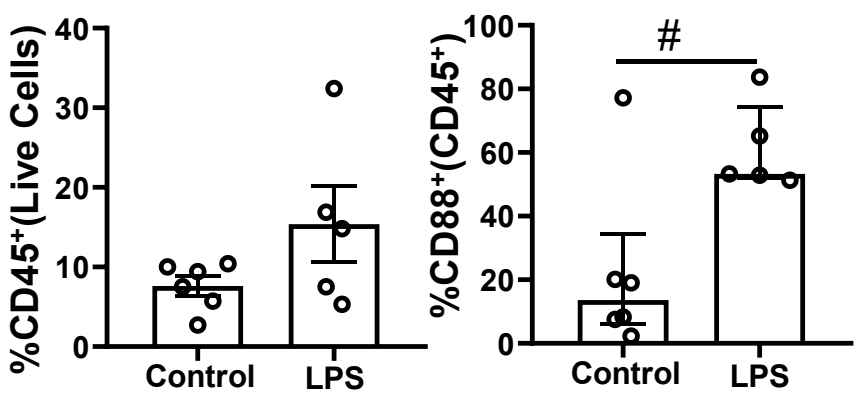

Fig. 2. Single cell RNAseq analysis of the fetal lung 16 hours post IA LPS exposure. The left lobe of fetal lung was processed into single cell suspensions and submitted for single cell sequencing. (A) UMAP from combined control and LPS-exposed animals (2 each). (B) Control (left) and IA LPS (right) exposed animals UMAP of re-clustered immune cell populations. (C) Representative dot plot of canonical cell type markers used in the identification of hematopoietic populations. (D) Feature plot of CD88(C5AR1) expression across hematopoietic cell populations and (E) percentage of total $C D 45^{+}$within live cells and $\mathrm{CD} 88^{+}$within $\mathrm{CD} 45^{+}$cells in lung. Data presented as mean with SEM, student's unpaired t-test $\left(\mathrm{CD} 45^{+} \%\right)$ or median and interquartile range, Mann-Whitney $U$ test $\left(C D 88^{+} \%\right) ; \# 0.05 \geq p \leq 0.10$. 
Fig. 4

A

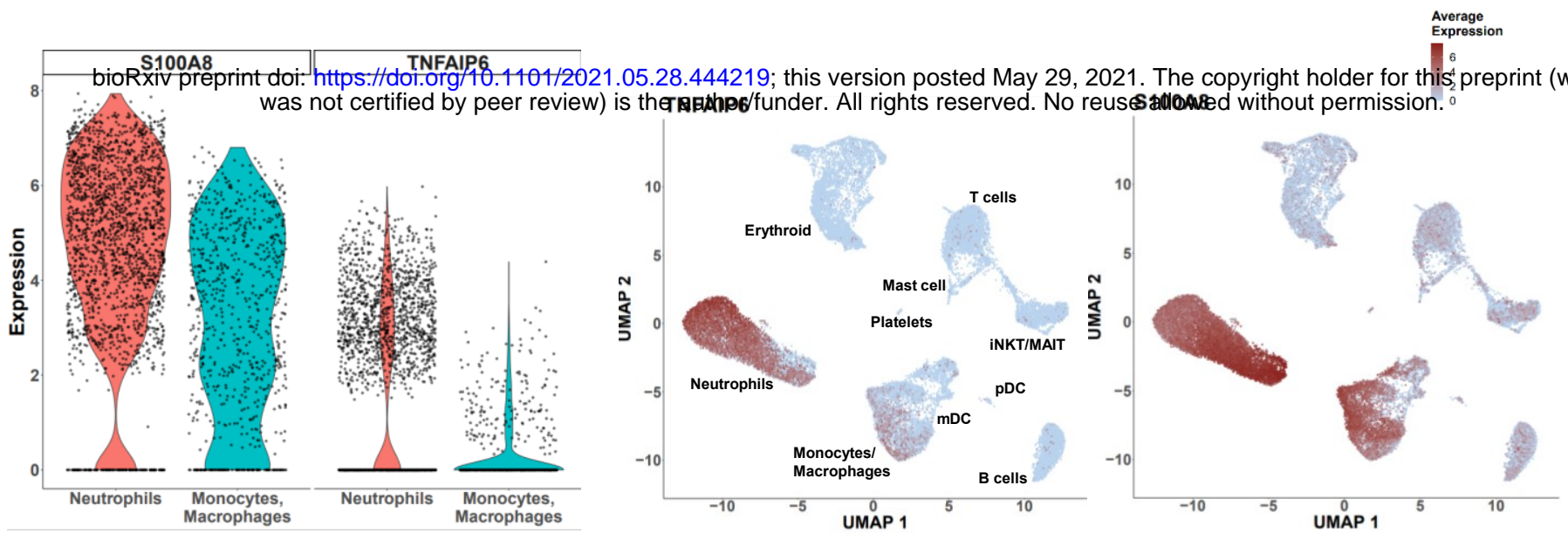

B

TNFAIP6-AF647

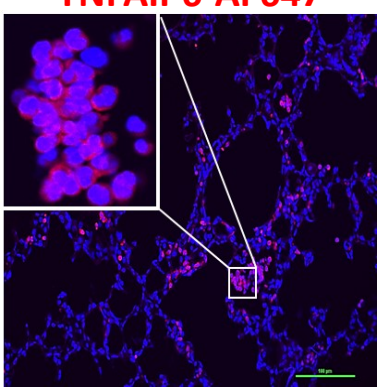

S100A8-FITC

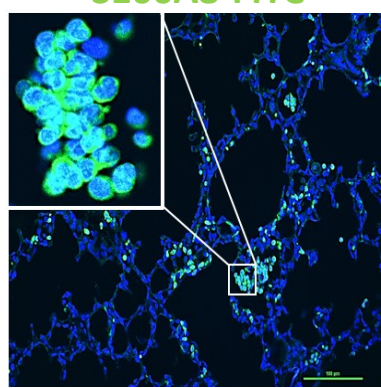

Overlay

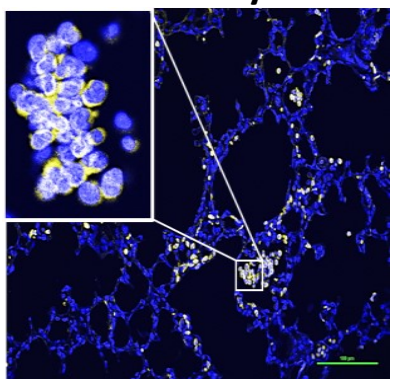

LPS
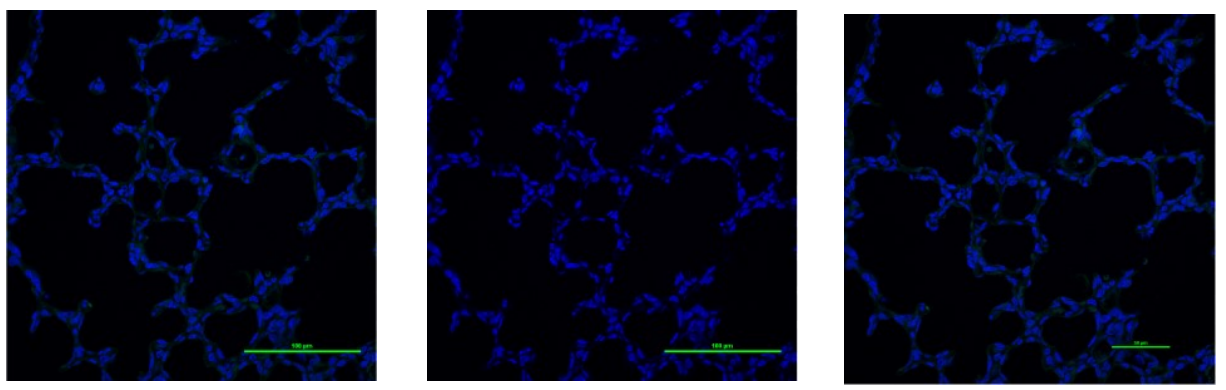

\section{Control}

C

D

Fig. 4. Fetal lung neutrophil response to IA LPS. (A) Violin (left) and feature (right) plot of TNFIAP6 and S100A8 expression in fetal lung neutrophils and monocyte/macrophage population following IA LPS exposure. (B) Immunofluorescence of IA LPS exposed fetal lung stained for S100A8 and TNFAIP6; scale bar is $100 \mu \mathrm{m}$. (C) Neutrophil aggregate count (left) and area (right) in the fetal lungs of control and IA LPS fetuses. Data presented as median and interquartile range, Mann-Whitney $U$ test; ${ }^{* *} p \leq 0.01$. (D) ssGSEA analysis of enriched GO terms in neutrophils. Numbers in parentheses represent the total number of genes for each process. 
Fig. 5

A

bioRxiv preprint doi: https://doi.org/10.1101/2021.05.28.444219; this version posted May 29, 2021. The copyright holder for this preprint ( was not certified by peer review) is the author/funder. All rights reserved. No reuse allowed without permission.

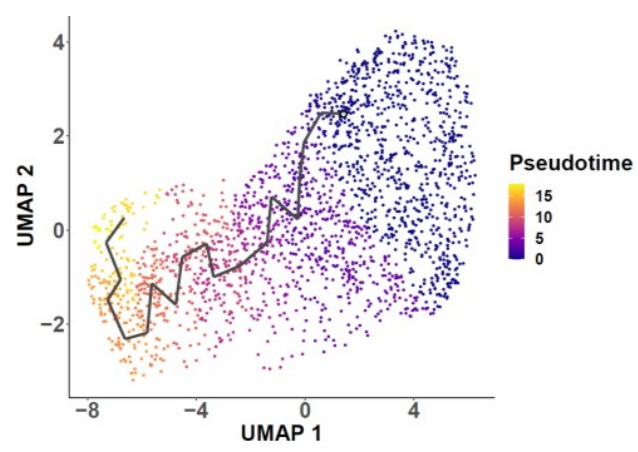

B

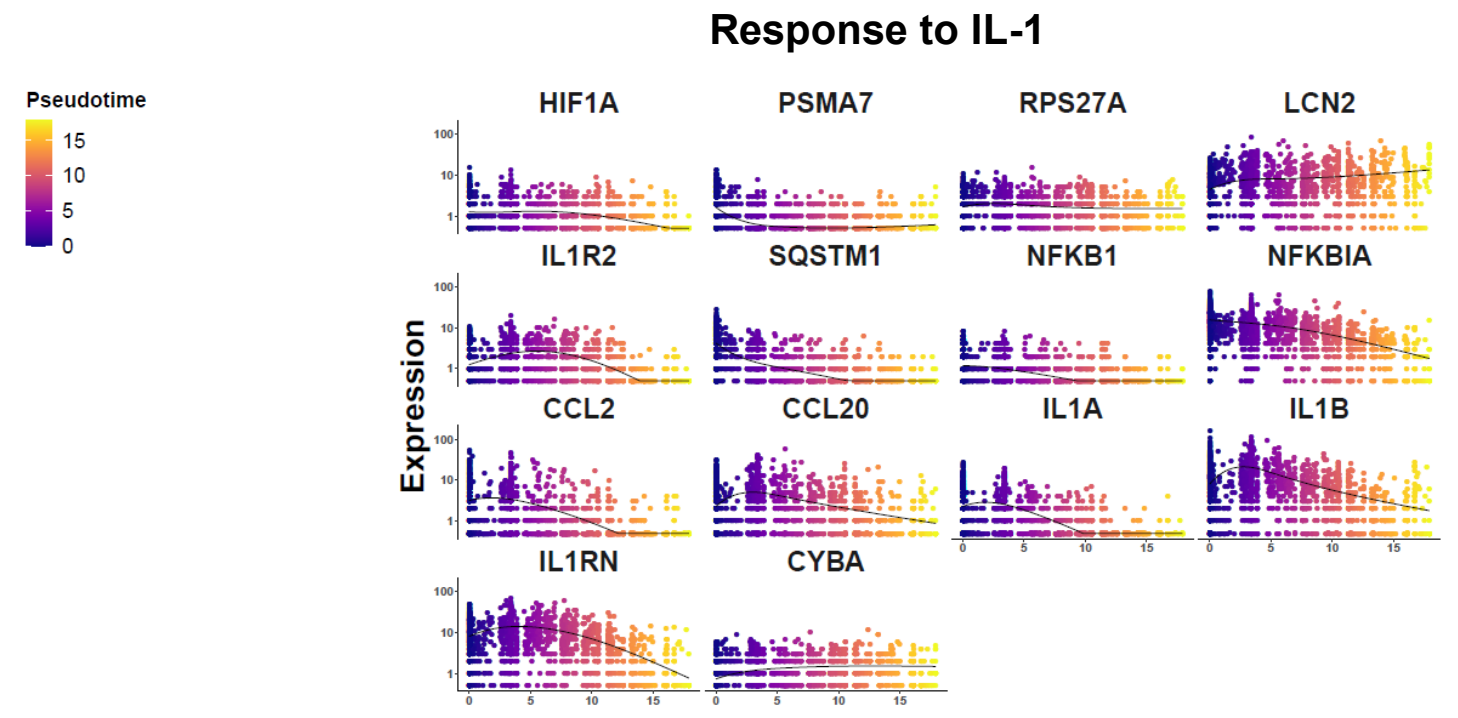

TLR Receptor Signaling Pathway

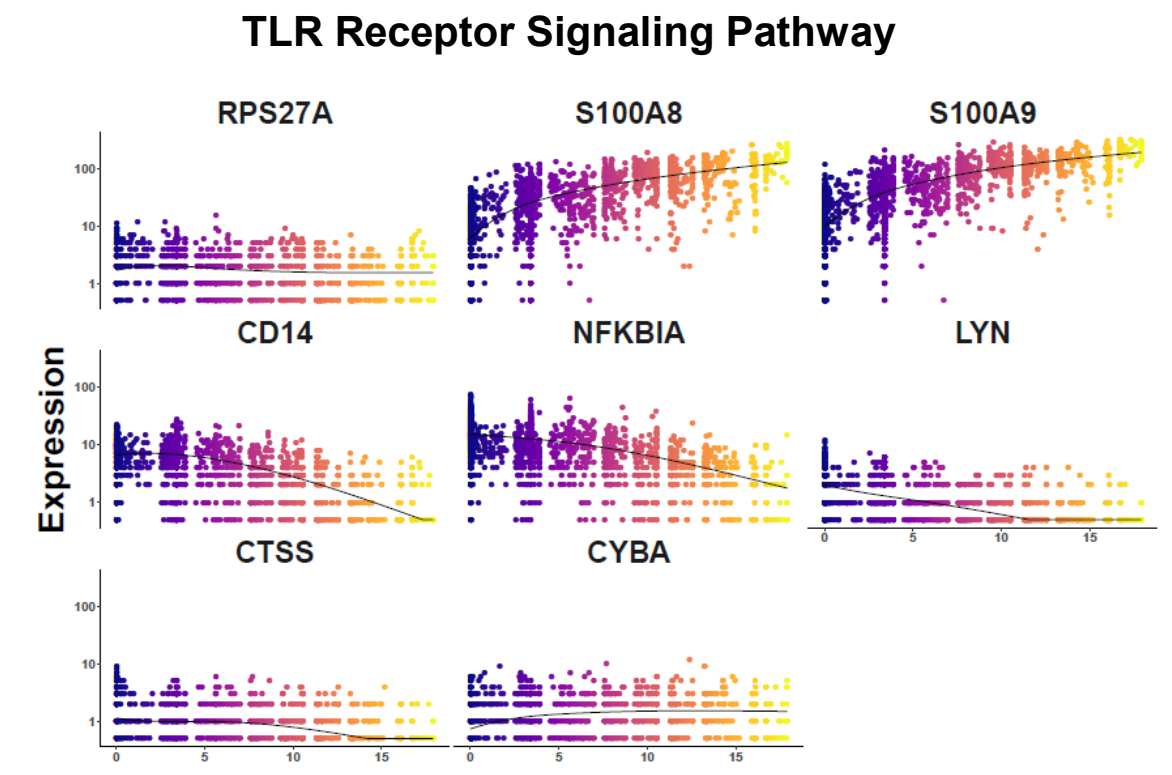

Fig. 5. Pseudo-time analysis of fetal lung neutrophil response to IA LPS. (A) Pseudo-time trajectory analysis of IA LPS exposed fetal lung neutrophils using Monocle. (B) Plots of genes from GO biological pathways response to IL-1 and TLR signaling pathway across pseudo-time. 
Fig. 6

A
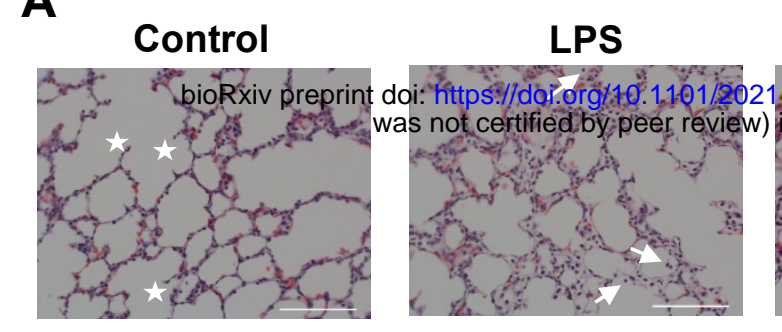

\section{LPS+IL-1RA}

LPS+aTNF

LPS+IL-1RA+aTNF

B
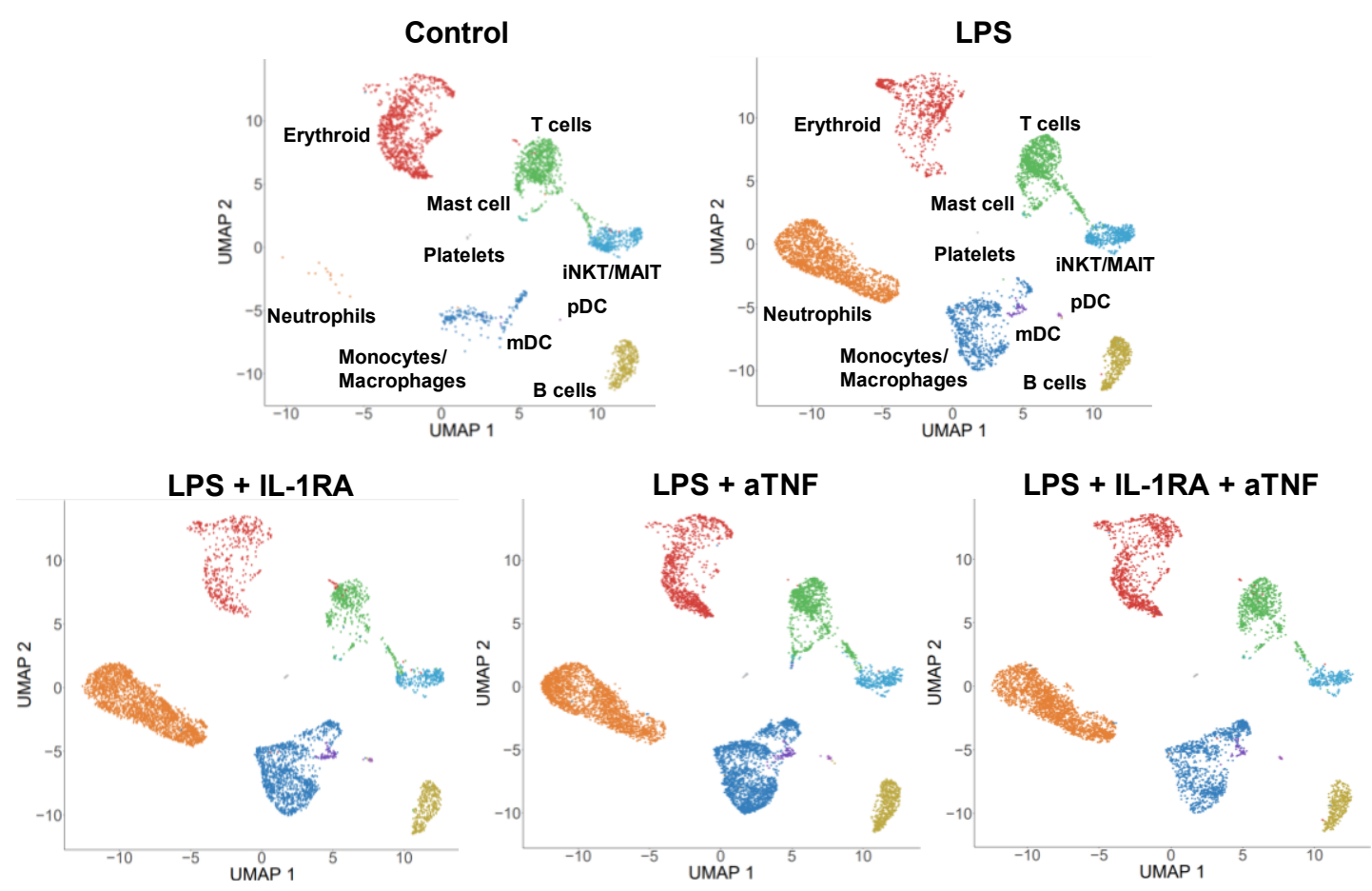

Erythroid $B$ cells Mast cells Monocytes, Macrophages $\mathrm{pDC}$
Neutrophils T cells iNKT/MAIT $\mathrm{mDC}$

C

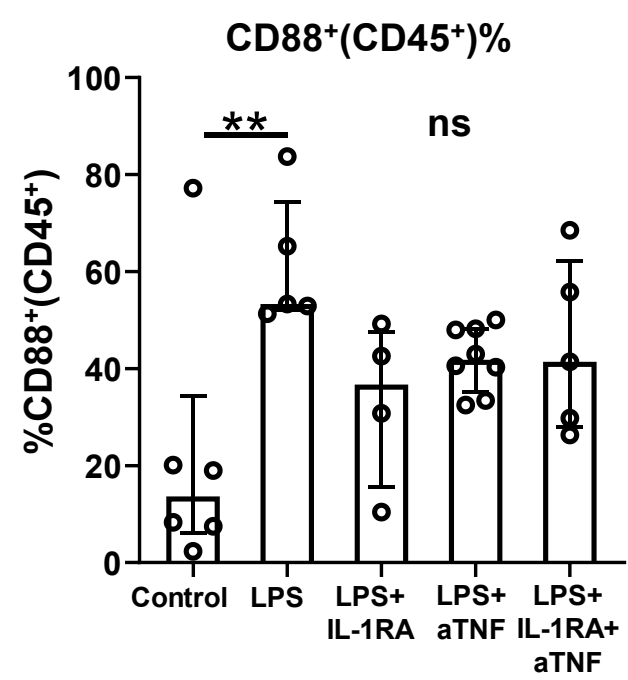

Fig. 6. Hematopoietic cell changes in the fetal lung following blocking IL-1 and TNF signaling. (A) 20x H\&E sections of fetal control, Ips, Ips+IL-1RA, Ips+anti-TNF, and Ips+IL-1RA+anti-TNF exposed animals; stars denote secondary septa, arrows represent cells in alveolar space and scale bar is $100 \mu \mathrm{m}$. (B) UMAPs of the hematopoietic cells from control, Ips, Ips+IL-1RA, Ips+anti-TNF, and Ips+ IL-1RA+antiTNF exposed animals. (C) $\mathrm{CD}^{+} 8^{+}$percentage within the $\mathrm{CD} 45^{+}$cells. Data presented as median and interquartile range, Kruskai-Wallis test; ${ }^{* *} p \leq 0.01$. 
bioRxiv preprint doi: https://doi.org/10.1101/2021.05.28.444219; this version posted May 29, 2021. The copyright holder for this preprint (n was not certified by peer review) is the author/funder. All rights reserved. No reuse allowed without permission.

Fig. 8

A

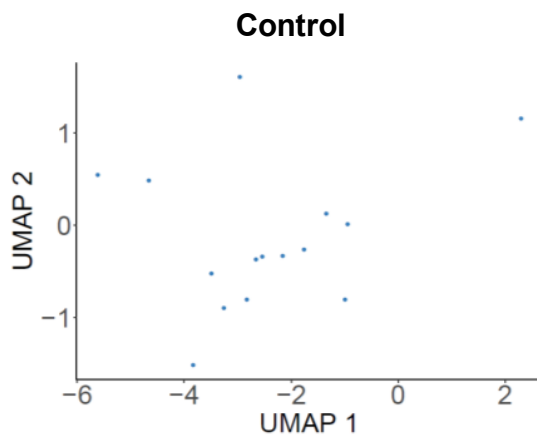

LPS

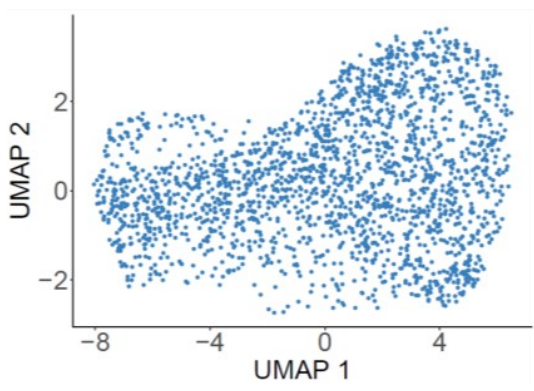

LPS+IL-1RA

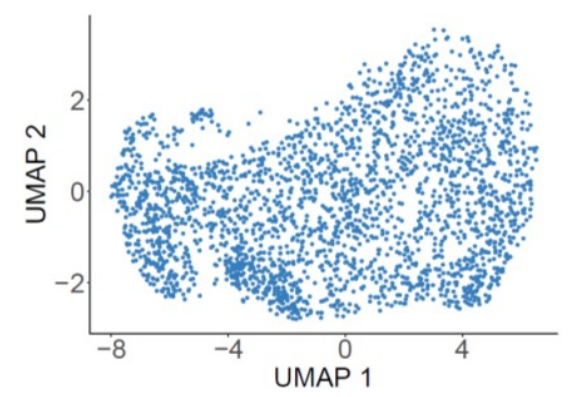

B

TLR receptor signaling pathway

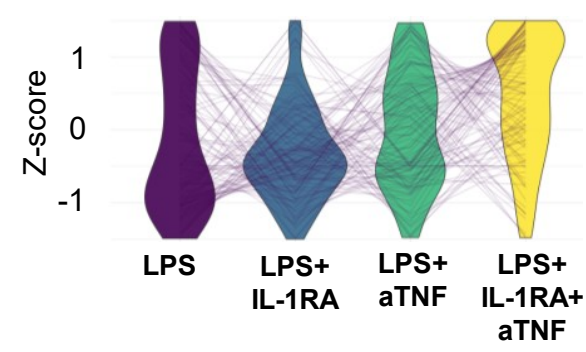

LPS+aTNF

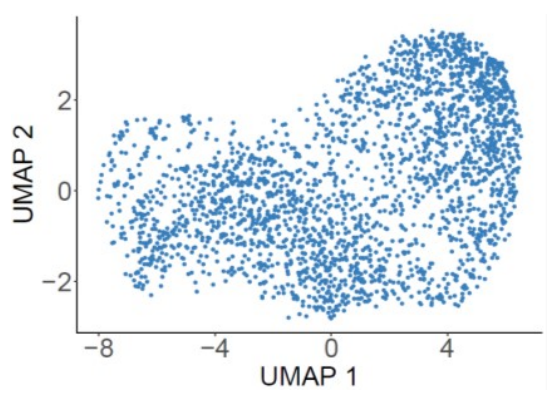

Response to IL-1

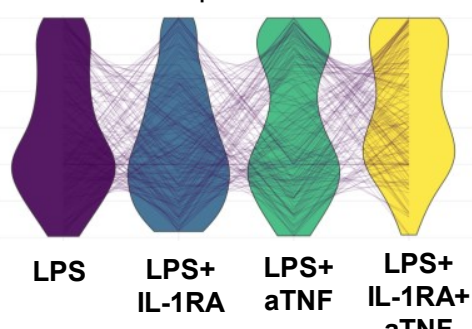

LPS+IL-1RA+aTNF

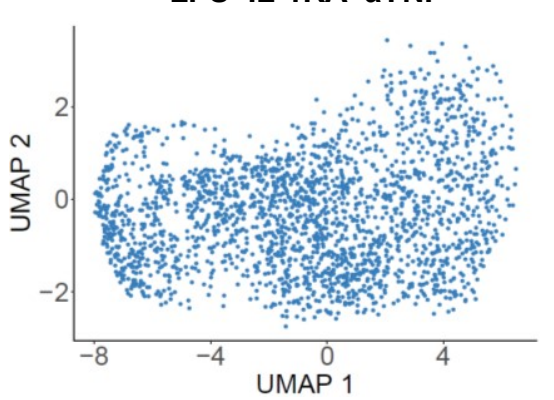

Response to TNF

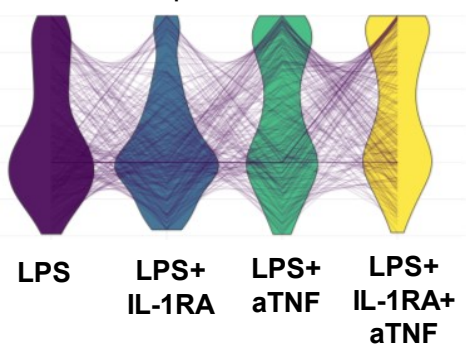

Fig. 8. Fetal lung neutrophils in response to IA LPS and blockades. (A) UMAPs of the neutrophil population across treatment conditions. (B) Parallel coordinate plots of scaled expression of representative genes in select biological processes across treatment conditions. 